\title{
The anatomical, physiological, and molecular analysis of a chlorophyll-deficient mutant in tree peony (Paeonia suffruticosa)
}

\author{
Q.S. CHANG ${ }^{*, \#, ~ L . X . ~ Z H A N G ~}{ }^{* *, \#,+}$, X.G. HOU ${ }^{* *}$, Z. WANG ${ }^{*}$, N. WANG*, M.G. GONG ${ }^{* * *}$, \\ Q.M. ZHANG ${ }^{*}$, H. CHEN*, Z.Q. SHI*, and C.C. DENG ${ }^{*}$ \\ College of Forestry, Henan University of Science and Technology, 471023 Luoyang, China* \\ College of Agriculture, Henan University of Science and Technology, 471023 Luoyang, China** \\ College of Food and Bioengineering, Henan University of Science and Technology, 471023 Luoyang, China***
}

\begin{abstract}
Tree peony is a famous ornamental plant in the world. However, little is known about the leaf color mutants in tree peony. The present study monitored the physiological and photosynthetic properties of a yellow leaf mutant (yll) in tree peony. The results showed that the $y l l$ mutant had lower pigment contents, but increased chlorophyll (Chl) $a / b$ and carotenoids to $\mathrm{Chl}$ ratio. Microstructure and ultrastructure analysis showed that the $y l l$ mutant had smaller chloroplasts, few thylakoid stacks, and a few stroma thylakoid membranes remained along with clusters of osmiophilic granules, which might result from inhibition at the reactions from coproporphyrinogen III to protoporphyrin IX. The yll mutant had lower leaf net photosynthetic rate, stomatal conductance, transpiration rate, and stomata limitation value, but higher intercellular $\mathrm{CO}_{2}$ concentration. Analysis of simple sequence repeat markers indicated that four pairs of primers could obtain different bands in the genome of the $y l l$ mutant compared to the wild type.
\end{abstract}

Additional key words: chlorophyll precursor; chlorophyll synthesis; gas exchange; net photosynthetic rate; porphobilinogen; protochlorophyllide.

\section{Introduction}

Chlorophylls (Chl) play a key role in the absorption, transfer, and conversion of light energy in photosynthetic organisms (Wang et al. 2018). Chl biosynthesis involves a series of enzymatic steps from glutamyl-tRNA to Chl $b$ (Nagata et al. 2005, Wu et al. 2007, Wang et al. 2014). Chl is synthesized in the grana of thylakoids, which assembles with Chl-binding proteins, and is inserted into thylakoid membranes of chloroplasts. The synthesis of Chl and plastid development is interdependent and inseparable (Yang et al. 2012, Wang et al. 2018). There are about 3,000 proteins in the chloroplast (Timmis et al. 2004, Reumann et al. 2005); these proteins play important roles in the transition from proplastids to mature chloroplasts, and some of them are involved in the synthesis and regulation of photosynthetic system, etc. (Sakamoto et al. 2008, Dong et al. 2013). There is no doubt that chloroplasts are important organelles for plant photosynthesis (Zhao et al. 2012).

Leaf color mutant is a valuable material in scientific research; it has been applied widely in chloroplast development research (Li et al. 2014, Zhang et al. 2017), Chl biosynthesis pathway (Yang et al. 2012, Chen et al. 2013), photosynthetic regulation (Wu et al. 2014, Yang et al. 2018), genetic breeding of higher plants, and other fields. Leaf color mutants have been identified in various plants, and most of them have been characterized in woody and cereal crops including tea (Wang et al. 2014), rice (Wu et al. 2014, Zhao et al. 2014, Wang et al. 2015), wheat (Wang et al. 2018), etc. So far, a lot of work has been done on the mechanism of leaf color variation. It is generally believed that the block of chloroplast development and Chl synthesis or degradation pathways in plant leaves lead to changes in leaf color (Zhao et al. 2016). It was reported that a rice (Oryza sativa) leaf color mutant ( $y g l l$ ) exhibited a yellow-green leaf phenotype due to a decrease of Chl synthase (Wu et al. 2007). The virescent yellowleaf mutant $(v y l)$ in rice showed reduced $\mathrm{Chl}$ and impaired chloroplast development, the $v y l$ gene mutation encoded a protein homologous Arabidopsis ClpP6, leading

Received 19 September 2018, accepted 20 February 2019.

${ }^{+}$Corresponding author; e-mail: hkdzlx@126.com

Abbreviations: ALA - delta-aminolevulinic acid; $\mathrm{Car}$ - carotenoids; $\mathrm{Chl}$ - chlorophyll; $C_{\mathrm{i}}$ - intercellular $\mathrm{CO}_{2}$ concentration; coprogen III coproporphyrinogen; $E$ - transpiration rate; $g_{\mathrm{s}}$ - stomatal conductance; $\mathrm{L}_{\mathrm{s}}$ - stomatal limitation; Mg-Proto IX - Mg-protoporphyrin IX; PBG - porphobilinogen; Pchlide - protochlorophyllide; $P_{\mathrm{N}}$ - net photosynthetic rate; Proto IX - protoporphyrin IX; SSR - simple sequence repeat; urogen III - uroporphyrinogen III.

Acknowledgments: This research has been supported by the Natural Science Foundation of Henan Province (182300410082, 182300410092, 182300410050, 182300410017); National Natural Science Foundation of China (Grant No. 31870093, 31800096); Scientific and Technological Research Projects in Henan province (162102110095, 182102110401); Key project of Higher Education of Henan Province (15A180037, 16A220005); Student research training program (SRTP) project of Henan University of Science and Technology $(2018396,2017335)$.

"These authors contributed equally to this work. 
to premature termination of the predicted gene product, loss of the conserved catalytic triad (serine-histidineaspartate) and the polypeptide-binding site of VYL, which is a virescent yellow leaf $(v y l)$ mutant in rice (Oryza sativa) (Dong et al. 2013). The yellow-leaf tea cultivar Zhonghuang 2 ( $\mathrm{ZH} 2)$ displayed a chlorotic phenotype with lower $\mathrm{Chl}$ contents and abnormal chloroplast ultrastructure, indicating that $\mathrm{Chl}$ biosynthesis was partially inhibited (Wang et al. 2014). The pylm mutant in Pak-choi (Brassica rapa) seedlings had yellow leaves with a reduced $\mathrm{Chl}$ content, loose lamellae structure of grana and lesser thylakoid accumulation, which was blocked in Chl $a$ production step of Chl biosynthesis (Zhang et al. 2017). Due to the partial block in the step of Mg-protoporphyrin IX synthesis, the yellow-green mutant Jimai5265yg in wheat had spherical chloroplasts with few plastoglobuli, lower Chl content with a higher $\mathrm{Chl} a / b$ ratio (Wang et al. 2018).

Leaf color mutants or Chl-deficient mutants are usually characterized by visible leaf color such as albino, xanthan, virescent, and so on. Among them, yellowing of leaves is an important type of mutation. In general, yellow leaf mutants occur due to the decrease in leaf $\mathrm{Chl}$ content $(\mathrm{Ou}$ et al. 2008, Zhang et al. 2017), accompanied by destruction of leaf thylakoid structure (Chen et al. 2008, Wang et al. 2013), reduction of the net photosynthetic rate and plant growth potential (Zhu et al. 2014a, Zhang et al. 2017, Yang et al. 2018). The mutations causing a typical yellow Chl-less mutant phenotype has also the effects on ratio between two photosystems (Terao and Katoh 1996, Brestič et al. 2015), which influences the photoprotective capacity of the mutants and tolerance to stress conditions (Georgieva et al. 2003, Brestič et al. 2016, Živčák et al. 2019).

In recent years, some leaf color mutants have been gradually discovered on some ornamental plants, such as Epipremnum aureum (Hung and Xie 2009), Chrysanthemum (Chang et al. 2013), Ulmus pumila (Zhu et al. 2014b), Anthurium andraeanum (Yang et al. 2015) and so on. However, there have been relatively few studies on leaf color mutants in flower plants. Tree peony (Paeonia suffruticosa) is one of famous traditional flower plants in China, and it is also well known in the world. It is an ornamental flowering plant whose floral organs are mainly ornamental objects. However, its flower has a short flowering period of only about seven days (Zhou 2015). At present, it is very difficult to extend the flowering period of tree peony, while the leaf has a longer life cycle, the discovery of peony leaf-viewing varieties can make up for the shortage of short flowering period of peony. and increase the ornamental value of tree peony. Although the physiological characteristics of leaf color mutants in many plants have been studied, little is known about the physiological mechanism of leaf color mutation in tree peony.

Tree peony yellow leaf variety 'Wu Long Peng Sheng' designed $y l l$ is a spontaneous mutant with a great ornamental and commercial value. In the present study, we performed the comparison analysis on the Chl content, Chl synthesis precursors, microstructure and ultrastructure, photosynthetic parameters, and amplified PCR products from total genomic DNA between the normal green leaf (wild type) and yellow leaf (yll mutant) in tree peony. We believe that the results of this study will help to provide basic data for the directional breeding of leaf-viewed tree peony cultivars.

\section{Materials and methods}

Material description: Wild type and natural yellow leaf mutant, $y l 1$, (Fig. 1A) of $P$. suffruticosa cultivar 'Wu Long Peng Sheng', were planted on the campus farm of

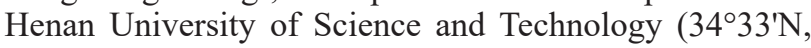
$112^{\circ} 16^{\prime} \mathrm{E}$ ), about $250 \mathrm{~m}$ above sea level, annual average temperature of $14.1^{\circ} \mathrm{C}$, annual average rainfall of $595 \mathrm{~mm}$; the soil is mainly cinnamon soil; soil profile structure is organic matter accumulation layer,viscosity layer, calcium layer, and parent layer (Chen and Yuan 2018). Tree peony was about 12 years old; both types of tree peony plants were planted by mean of random design and maintained in natural conditions under direct sunlight for normal field management. Green leaves (Fig. 1B) from wild type plants and yellow leaves (Fig. 1C) from yll mutants were selected for experimental analysis in April 2017.

Measurement of pigment content: Fresh leaf samples $(0.10 \mathrm{~g})$ were cut into thin strips, extracted with a mixed solution of $5 \mathrm{ml}$ of acetone and water $(4: 1, \mathrm{v} / \mathrm{v})$ for $48 \mathrm{~h}$ under dark conditions. The extraction was then mixed, and the absorbance of the supernatants was recorded at 470, 646, and $663 \mathrm{~nm}$ (752s, Shanghai Lengguang Technology Co., Ltd., China) Pigment content (Chl $a$, Chl $b$, and Car) was determined spectrophotometrically from four biological replicates per treatment following the method of Zhang and Fan (2007).

Measurement of Chl precursors: The Chl synthesis precursor delta-aminolevulinic acid (ALA) was extracted as described by Dei (1985). The isolation of porphobilinogen (PBG), uroporphyrinogen III (urogen III) and coproporphyrinogen III (coprogen III) was performed using the method of Bogorad (1962), while protoporphyrin IX (Proto IX), Mg-protoporphyrin IX (Mg-Proto IX), and protochlorophyllide (Pchlide) were determined according to the method of Rebeiz et al. (1975). Each index was subjected to three biological repetitions. The concentration of the Chl precursor from the wild type plant was set to $100 \%$.

Leaf microstructure and ultrastructure analysis: Leaf samples were taken from the developing leaves, cut into about $5-\mathrm{mm}^{2}$ segments, and fixed in formalin:alcohol:glacial acetic acid (90:5:5 by volume) for at least $24 \mathrm{~h}$. The fixed leaves were dehydrated through an ethanol series, cleared in xylene for two times, then embedded in paraffin wax according to the method of Deng et al. (2012), sliced into transverse $8-\mu \mathrm{m}$ thick sections using a rotary microtome (Leica 2016, Germany). These sections were doubly stained with safranin and Fast Green, mounted in Canada balsam. Photographs were obtained by using a Leica microscope (DM 2500, Leica Corp., Wetzlar, Germany). 
The developing leaves from wild type and the $y l l$ mutant plants were subjected to transmission electron microscopy analysis according to the method of Teng et al. (2006). A $1 \times 1 \mathrm{~mm}$ leaf segment of tree peony was independently fixed for $24 \mathrm{~h}$ at $4{ }^{\circ} \mathrm{C}$ in $2.5 \%(\mathrm{w} / \mathrm{v})$ glutaraldehyde and $1 \%(\mathrm{v} / \mathrm{v})$ formaldehyde in $0.1 \mathrm{M}$ phosphate buffer $(\mathrm{pH} 7.4)$, followed by fixation at $4{ }^{\circ} \mathrm{C}$ in $1 \%(\mathrm{w} / \mathrm{v}) \mathrm{OsO}_{4}$ for $2 \mathrm{~h}$. The fixed tissue was dehydrated through a graded series of ethanol and acetone, then replaced twice with propylene oxide, and finally embedded in epoxy resin. After ultrathin sectioning, the samples were stained with $1 \%(\mathrm{w} / \mathrm{v})$ aqueous uranyl acetate and lead citrate solutions. The images were observed by a transmission electron microscope (H7650, Hitachi, Japan).

Gas-exchange parameters: Net photosynthetic rate $\left(P_{\mathrm{N}}\right)$, stomatal conductance $\left(g_{\mathrm{s}}\right)$, intercellular $\mathrm{CO}_{2}$ concentration $\left(C_{\mathrm{i}}\right)$, and transpiration rate $(E)$ were measured by a portable photosynthesis system (LI-6400, LICOR, Lincoln, NE, USA) in the sunny morning between 9:00-11:00 h, the $\mathrm{CO}_{2}$ concentration was $380 \mu \mathrm{mol} \mathrm{mol}{ }^{-1}$, the leaf chamber temperature was $25^{\circ} \mathrm{C}$, and the PPFD was $1,200 \mu \mathrm{mol}$ $\mathrm{m}^{-2} \mathrm{~s}^{-1}$ in the sample chamber. Gas-exchange parameters were measured on the full-expanded third or fourth leaf (from top). Stomatal limitation value $\left(\mathrm{L}_{\mathrm{s}}\right)$ was calculated according to Berry and Downton (1982) using the formula: $\mathrm{L}_{\mathrm{s}}=1-C_{\mathrm{i}} / C_{\mathrm{a}}$. Each material was repeated four times.

SSR analysis: The fully expanded leaf tissues were used for total DNA extraction using the CTAB method (Guo 2016). DNA quality was evaluated by $1.2 \%$ agarose gel electrophoresis, and then maintained at $-80^{\circ} \mathrm{C}$. DNA was quantified by a spectrophotometer ND-1000 (Nanodrop Technologies, Wilmington DE). Sixteen pairs of SSR (simple short repeats) markers from Hou et al. (2011) and Wang (2011) were utilized for SSR analysis. The reaction volume was $20 \mu \mathrm{L}$ and contained: $60 \mathrm{ng}$ DNA templates, $1 \mathrm{U}$ Taq polymerase (TaKaRa, Dalian, China), $0.6 \mathrm{mM}$ dNTPs, $0.5 \mu \mathrm{M}$ forward and reverse primers, $1 \times$ Taq buffer $\left(\mathrm{Mg}^{2+}\right)$, and distilled water. The initial denaturing step was $94^{\circ} \mathrm{C}$ for $5 \mathrm{~min}, 35$ cycles of denaturation for $45 \mathrm{~s}$ at $94^{\circ} \mathrm{C}$, annealing for $45 \mathrm{~s}$ at $50^{\circ} \mathrm{C}$ and $60 \mathrm{~s}$ at $72^{\circ} \mathrm{C}$, and a final extension at $72^{\circ} \mathrm{C}$ for $8 \mathrm{~min}$. The amplified products were separated on a $6 \%$ polyacrylamide denaturing gel and visualized by silver staining.

Statistical analysis: The data in the tables and figures are expressed as the means \pm standard deviation (SD) from at least three independent biological replicates. Student's $t$-test was employed with SPSS 16.0 to determine the difference between wild type and $y l l$ mutant plants.

\section{Results}

Pigment measurements: The $y l l$ mutant is a natural mutant, which is of a yellow leaf phenotype, and is different from the surrounding wild type plants (Fig. 1A). Leaf pigment measurement showed that the Chl content in the $y l 1$ mutant was greatly reduced by $89.8 \%$ in $\mathrm{Chl} a$, by $94.8 \%$ in Chl $b$, and by $91.1 \%$ in total Chl (Table 1 ).
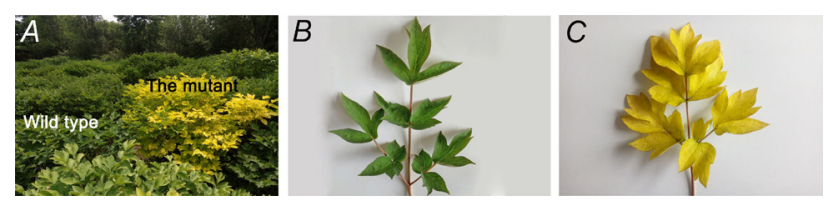

Fig. 1. Phenotypic characterization of wild type and the $y l l$ mutant in tree peony. $(A)$ wild type (left), the mutant (right). $(B)$ green leaf of the wild type. (C) yellow leaf of the mutant.

The Car content also decreased significantly compared with wild type plants. It is worth mentioning that both Chl $a / b$ and Car/Chl increased remarkably, 1.98-fold and 4.81-fold that of wild type plants, respectively.

Leaf microstructure and ultrastructure: There was no obvious difference between the green leaf and yellow leaf internal structure. Both leaves had visible upper epidermis, palisade tissue, sponge tissue, and lower epidermis. In the green leaves of wild type plants, most of the chloroplasts were presented in mesophyll cells, which were composed of palisade mesophyll cells and spongy mesophyll cells (Fig. 2A). The number of chloroplasts in palisade tissue was higher than that in spongy tissue. The leaf microstructure in yellow leaves was similar to that of the green leaves (Fig. 2B), but the space of sponge tissue was larger than that of the wild type plants. The yellow leaf section was lighter in color, and the smaller size of chloroplasts in the yellow leaf might be one of the reasons. These results could be confirmed in the ultrastructure of the chloroplast in yellow leaf (Fig. 3A,D). Meanwhile, less Chl might be also another reason for its lighter color.

There was a significant difference in chloroplast ultrastructure between the green leaf of the wild type plant and the yellow leaf of the $y l l$ mutant (Fig. 3). The chloroplasts of the wild type plants had highly organized inner membrane system, and many granal thylakoids were regularly distributed with plentiful granal lamellae, the osmiophilic granules were dispersed and lesser in number (Fig. 3A-C). In contrast, the stacks of grana disappeared from the chloroplasts of the yellow leaves in the $y l l$ mutant (Fig. 3D-F), and only a few stromal thylakoid membranes remained along with clusters of osmiophilic granules. The structure of thylakoid membranes in these chloroplasts was extremely disordered. Furthermore, the size of the chloroplast in the $y l l$ mutant was relatively smaller compared to the wild type plants.

Analysis of $\mathrm{Chl}$ precursors content: The relative content of seven precursors of $\mathrm{Chl}$ biosynthesis was tested in this study (Fig. 4). The relative contents of ALA, PBG, urogen III, and coprogen III in the $y l l$ mutant were significantly higher compared to the wild type plants. However, the contents of Proto IX, Mg-Proto IX, and Pchlide showed a sharp decrease, similar to the contents of Chl $a$ or Chl $b$.

Photosynthetic parameters analysis: The photosynthetic parameters of the $y l l$ mutant were much lower than those of the wild type plants (Table 2). In the $y l l$ mutant plants, $P_{\mathrm{N}}$, $g_{\mathrm{s}}, E$, and $\mathrm{L}_{\mathrm{s}}$ were reduced by $69.8,57.2,50.1$, and $31.5 \%$ 
Table 1. Pigment contents in the leaves of tree peony wild type and $y l l$ mutant, calculated per fresh mass $\left[\mathrm{mg} \mathrm{g}^{-1}\right]$. Different small letters in the same column mean significant difference at 0.05 level as determined by Student's t-test.

\begin{tabular}{lllllll}
\hline Plant type & Chl $a\left[\mathrm{mg} \mathrm{g}^{-1}\right]$ & $\mathrm{Chl} b\left[\mathrm{mg} \mathrm{g}^{-1}\right]$ & $\mathrm{Chl} a / b$ & $\mathrm{Car}\left[\mathrm{mg} \mathrm{g}^{-1}\right]$ & $\mathrm{Chl} a+b\left[\mathrm{mg} \mathrm{g}^{-1}\right]$ & $\mathrm{Car} / \mathrm{Chl}$ \\
\hline WT & $1.428 \pm 0.087^{\mathrm{a}}$ & $0.471 \pm 0.079^{\mathrm{b}}$ & $3.070 \pm 0.395^{\mathrm{a}}$ & $0.355 \pm 0.016^{\mathrm{a}}$ & $1.899 \pm 0.159^{\mathrm{a}}$ & $0.188 \pm 0.021^{\mathrm{b}}$ \\
$y l l$ & $0.145 \pm 0.003^{\mathrm{b}}$ & $0.024 \pm 0.004^{\mathrm{a}}$ & $6.063 \pm 0.939^{\mathrm{b}}$ & $0.153 \pm 0.009^{\mathrm{b}}$ & $0.170 \pm 0.007^{\mathrm{b}}$ & $0.903 \pm 0.084^{\mathrm{a}}$ \\
\hline
\end{tabular}
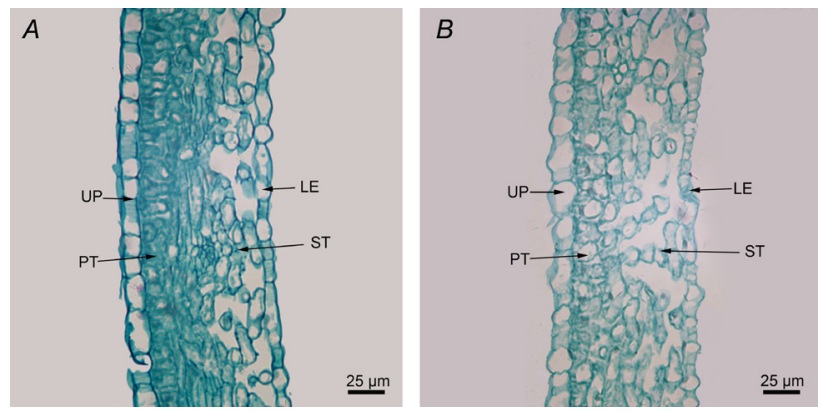

Fig. 2. Anatomical structures of green leaf in wild type $(A)$ and yellow leaf in peony $y l l$ mutant $(B)$. LE - lower epidermis; $\mathrm{PT}$ - palisade tissue; ST - spongy tissue; UP - upper epidermis.

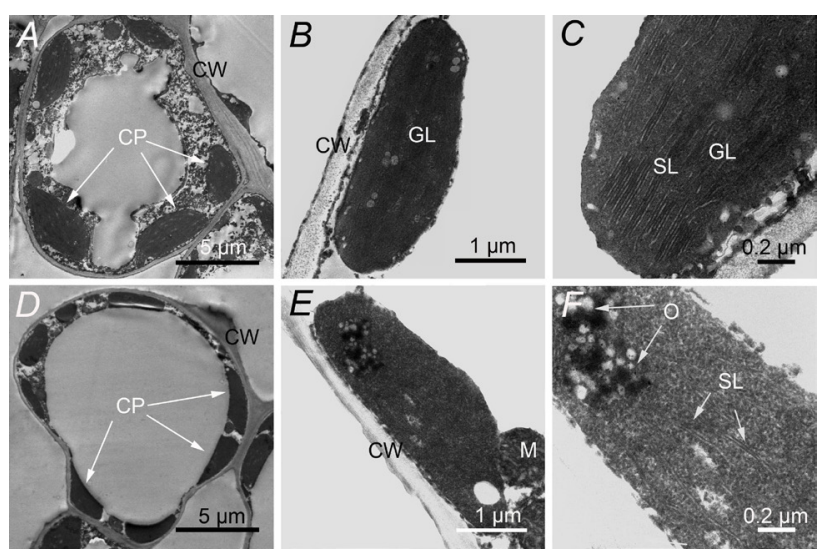

Fig. 3. Chloroplast ultrastructure of the green leaves in wild type $(A-C)$ and yellow leaves in the $y l l$ mutant $(D-F) . \mathrm{CP}$ - chloroplast; $\mathrm{CW}$ - cell wall; GL - granum lamella; $\mathrm{M}$ - mitochondrion; $\mathrm{O}$ osmiophilic granules; SL - stroma lamella.

compared to that in the wild type plants, respectively. In contrast, $C_{\mathrm{i}}$ in the $y l l$ mutant was $15.1 \%$ higher than that in the wild type plants.

SSR analysis: Sixteen pairs of SSR primers were used to amplify PCR products from total genomic DNA of tree peony (Table 3 ). As could be seen from the Fig. 5, the selected primers could obtain clear amplified bands. Of the 16 primer combinations tested, four pairs $(24 \mathrm{f} / \mathrm{z}$, 26f/z, PAC51, PAC78) of them produced some differences in band intensity or size between wild type and yll mutant plants, other combinations showed no significant variation.

In view of the differential bands produced by four pairs of primers, it is suggested that there were some genetic differences in DNA levels between wild type and yll mutant plants. It could be preliminarily determined that the $y l l$ mutant was produced by changes in genetic material of wild type plant.

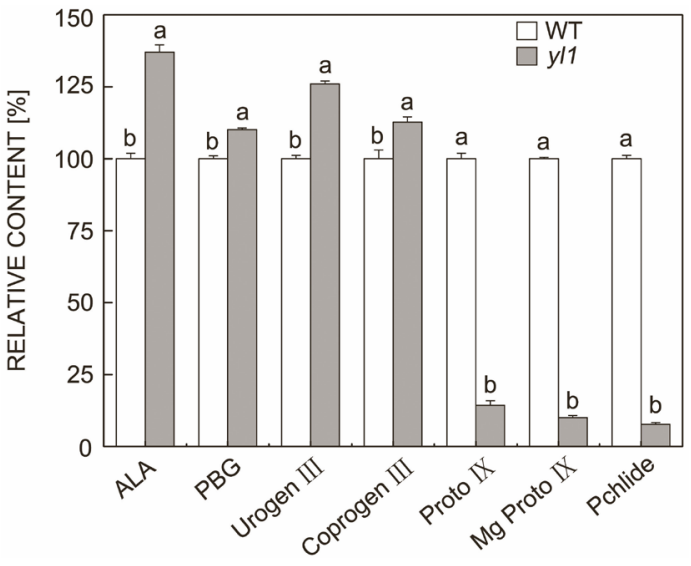

Fig. 4. Comparison of chlorophyll synthesis precursors between wild type and the $y l l$ mutant (every precursor's content of the wild type was set as $100 \%$ ). Different small letters mean significant difference at 0.05 level, respectively, as determined by the $t$-test.

\section{Discussion}

Leaf color mutants not only have important scientific research value, but also have important application value, especially leaf color mutants of flower plants (Yang et al. 2015, Cao et al. 2017). Tree peony leaf color mutants have drawn much attention due to their unique leaf color, economic and application value. In this study, we reported a novel Chl-deficient tree peony cultivar that exhibited a yellow leaf phenotype and performed the physiological and SSR analysis of this mutant (yl1).

Pigment analysis showed that the Chl $b$ content in the yellow leaf of $y l l$ mutant was markedly lower than that of the wild type, indicating that the reduction of $\mathrm{Chl} b$ was greater than that of $\mathrm{Chl} a$, and thus this mutant belonged to be a Chl $b$-deficient mutant. The Chl contents of the $y l l$ mutant greatly decreased compared to the wild type plants, but the ratio of $\mathrm{Car} / \mathrm{Chl}$ in yellow leaves of $y l l$ mutant was relatively higher than that of the wild type, and these results might be the direct cause of the yellow appearance of the $y l l$ mutant. At the same time, a significant increase in Chl $a / b$ of the $y l l$ mutant indicated that the light-harvesting antenna complexes and the photosystems were seriously damaged, which was consistent with the ultrastructure. Similar results were also found in the yellow leaf mutant pylm of Pak-choi (Brassica rapa) (Zhang et al. 2017).

In general, thylakoid membranes in higher plants are regularly arranged and stacked into the grana of chloroplasts, while many leaf color mutants often exhibited abnormal thylakoid structure, which also indicated a decrease in photosynthetic capacity (Wu et al. 2014, Zhu et al. 2014a, Zhang et al. 2017). In the yll mutant, the 
Table 2. Photosynthetic parameters in the leaves of the wild type and the $y l l$ mutant of tree peony. $P_{\mathrm{N}}-$ net photosynthetic rate; $g_{\mathrm{s}}$ - stomatal conductance; $C_{\mathrm{i}}$ - intercellular $\mathrm{CO}_{2}$ concentration; $E$ - transpiration rate; $\mathrm{L}_{\mathrm{s}}-$ stomatal limitation. Different small letters in the same column mean significant difference at 0.05 level as determined by Student's $t$-test.

\begin{tabular}{llllll}
\hline Plant type & $P_{\mathrm{N}}\left[\mu \operatorname{mol}\left(\mathrm{CO}_{2}\right) \mathrm{m}^{-2} \mathrm{~s}^{-1}\right]$ & $g_{\mathrm{s}}\left[\operatorname{mol}\left(\mathrm{H}_{2} \mathrm{O}\right) \mathrm{m}^{-2} \mathrm{~s}^{-1}\right]$ & $C_{\mathrm{i}}\left[\mu \mathrm{mol}\left(\mathrm{CO}_{2}\right) \mathrm{mol}^{-1}\right]$ & $E\left[\mathrm{~mol}\left(\mathrm{H}_{2} \mathrm{O}\right) \mathrm{m}^{-2} \mathrm{~s}^{-1}\right]$ & $\mathrm{L}_{\mathrm{s}}$ \\
\hline WT & $11.94 \pm 0.42^{\mathrm{a}}$ & $0.19 \pm 0.05^{\mathrm{a}}$ & $272.02 \pm 25.95^{\mathrm{b}}$ & $2.73 \pm 0.67^{\mathrm{a}}$ & $0.30 \pm 0.06^{\mathrm{a}}$ \\
$y l l$ & $3.60 \pm 0.20^{\mathrm{b}}$ & $0.08 \pm 0.01^{\mathrm{b}}$ & $313.06 \pm 7.13^{\mathrm{a}}$ & $1.36 \pm 0.24^{\mathrm{b}}$ & $0.21 \pm 0.02^{\mathrm{b}}$ \\
\hline
\end{tabular}

Table 3. Sixteen pairs of SSR primers in tree peony according to Hou et al. (2011) and Wang (2011).

\begin{tabular}{|c|c|c|c|}
\hline Name/Locus & Genbank No. & Primer $1\left(5^{\prime}-3^{\prime}\right)$ & Primer $2\left(5^{\prime}-3^{\prime}\right)$ \\
\hline $10 \mathrm{f} / \mathrm{z}$ & FE528847 & GACGGAGAGAAAGAGAGCATA & GACAAAGACTGACACAGCGAT \\
\hline $16 f / z$ & FE528353 & GCTCATTACCGCTACTACCA & AAAACCACTCACCTCCCA \\
\hline $18 \mathrm{f} / \mathrm{z}$ & FE528918 & GTTCATTTTCATTCGGGGAC & AACCAAGCCAACTCACG \\
\hline $23 \mathrm{f} / \mathrm{z}$ & FE527983 & GGCTAATCTTGTTGCTCAG & ААССССТСТТТСТССТСА \\
\hline $24 f / z$ & FE528215 & TACCCTCCCGCTCCTGTTA & AAATCGTGTAGTGCCCTCA \\
\hline $26 f / z$ & FE529419 & TAGCCGAAACAGCAAAGC & TTCTCATCCGTCCAAGTCCA \\
\hline $28 \mathrm{f} / \mathrm{z}$ & FE528396 & AAATACCACCTCCAGACCGA & СТCTTCACCTTGTTCCACG \\
\hline $29 f / z$ & FE528916 & CGAAGTAAAGAAACAAGCGTA & TAGCCTCTGGACCAACCT \\
\hline $7 f / z$ & FE529771 & CGCCAAACGAATGGTCTA & GATGAGTGAGTTGAGTAAGGG \\
\hline $9 f / z$ & FE528055 & GAGAGACCACTCAAAAGGAAT & TGGGGCAGATGCGATGT \\
\hline $12 \mathrm{f} / \mathrm{z}$ & FE528793 & ATGGCTTTGCTGGAGATA & AGAAGACAACCGCAGACGC \\
\hline PAC38 & GQ480167 & GATGGGTATGATTGTGAGCA & GTTCCTGTGGTTTGACTTTC \\
\hline PC2 & GQ480172 & AAATCACAACACTCCTCACC & CTTCTCCAGCGTAATCCATA \\
\hline PC6 & GQ480175 & TCTTTCCATTTTCATAGATTTT & CAAATAAACCAACACCATAAGA \\
\hline PAC51 & GQ480181 & AGAGATTGATGAGTCCTGAGTA & TGAAGGTTTGTAAAGTAGGAGA \\
\hline PAC78 & GQ480192 & CATCTTCACTACTATCCAGGTC & TTACCATAAGGATGATGATTCT \\
\hline
\end{tabular}

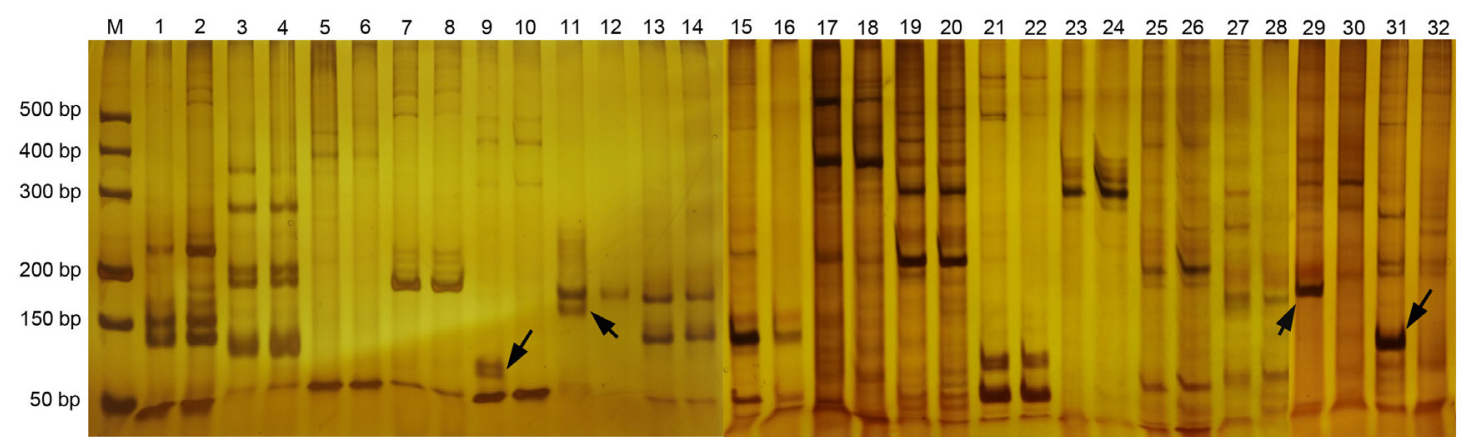

Fig. 5. The amplification and polymorphism of wild type and the $y l 1$ mutant. M - DNA marker with band sizes of 50, 150, 200, 300, 400, and $500 \mathrm{bp}$. DNA isolated from the green leaves of wild type (lanes 1, 3, 5, 7, 9, 11, 13, 15, 17, 19, 21, 23, 25, 27, 29, 31) and yellow leaves of the $y l 1$ mutant (lanes $2,4,6,8,10,12,14,16,18,20,22,24,26,28,30,32$ ) was amplified by PCR using the SSR primers from Hou et al.(2011) and Wang (2011).

chloroplast contained no grana stacks, fewer thylakoid lamellae, and clusters of osmiophilic granules. The efficient absorption and conversion of light in plants depends on the regular arrangement and stacking of the thylakoid membranes (Zhang et al. 2017). The lower photosynthetic parameters of the $y l l$ mutant were probably caused by the aberrant changes in chloroplast strutcture, which might also be due to a decrease in pigment contents. According to the microstructure and ultrastructure, the notable decrease in the $\mathrm{Chl}$ content of yellow leaves might be caused by the smaller chloroplasts and the absence of thylakoid membrane layers. Similar results were also found in chrysanthemum mutant (Chang et al. 2013) and rice ygll mutant (Wu et al. 2014) and other plants.

In the Chl synthesis pathway, many enzymes catalyze a series of enzymatic reaction steps. Analysis of Chl metabolic intermediates facilitates the analysis of blocking steps in Chl synthesis. In the chd6 mutant, some successive enzymes of $\mathrm{Chl}$ synthesis could be inhibited to varying degrees, severe inhibition occurred during the process of Mg-Proto IX to Pchlide or Mg-Proto IX to Chl $b$ (Yang et al. 2012). Compared with the control, the pylm 
mutant in Brassica rapa accumulated higher amounts of Mg-Proto IX, Pchlide, and Chlide, and lower Chl $a$ content. Therefore, it could be predicted that the blocking site of Chl biosynthesis in pylm mutant would be the step of esterification of Chlide to Chl a (Zhang et al. 2017). Compared with wild type plants, the $y l 1$ mutant accumulated higher contents of ALA, PBG, urogen III, and coprogen III, but lower amounts of Proto IX, Mg-Proto IX, and Pchlide. These results indicated Chl biosynthesis in the $y l l$ mutant might be blocked during the conversion of coprogen III to Proto IX.

Chl is mainly distributed in thylakoid membrane. The normal Chl content, the regular arrangement, and the stacking of thylakoids in the leaves can effectively ensure the absorption and transformation of light energy, so that photosynthesis can function normally. Studies have shown that abnormal structural changes of thylakoids in Chl-deficient mutants are often accompanied by loss of Chl (Zhu et al. 2014a, Zhang et al. 2017). The lower photosynthetic rate of the $y l 1$ mutant might be caused by the abnormal structure of thylakoid membranes and the significant decrease in Chl content.

In this study, it is interesting to note that if $P_{\mathrm{N}}$ was calculated as per the unit of Chl content, it would be higher in $y l l$ mutant than that in the wild type plant. Under conditions of equal Chl content, the $y l l$ mutant with higher $\mathrm{Chl} a / b$ contained more $\mathrm{Chl} a$ than that of wild type plant. It is well known that $\mathrm{Chl} a$ can convert light energy into chemical energy, while Chl $b$ only absorbs light energy (Pan 2004). Then, there could be no doubt that the higher contents of Chl $a$ in $y l l$ mutant could increase photosynthetic capacity more effectively than in the wild type. In addition, the chloroplast were located deep in the leaves of $y l l$ mutant with less Chl, which could theoretically obtain more penetration light, and an increased $C_{\mathrm{i}}$ value could provide a higher $\mathrm{CO}_{2}$ supply (Wang et al. 2018). Therefore, in the presence of a high proportion of $\mathrm{Chl} a$, the photosynthetic capacity of the yll mutant would be improved. Furthermore, it is easy to understand that the photosynthetic rate per Chl unit in $y l 1$ mutant was higher than that of the wild type, which might be a compensation for Chl deficiency in the $y l l$ mutant. Similar results were also found in yellow-green mutants of rice (Hu et al. 1981) and yellow-leaf mutant tobacco (Lei et al. 2010).

In this study, SSR analysis was used to identify DNA levels. In view of the differential bands produced by the four pairs of primers, there were some genetic differences in DNA levels $y l l$ mutant plants. It could be initially determined that the $y l l$ mutant was produced by changes in genetic material of wild type plant. Of course, further research is needed to clarify the cause of the mutation.

Conclusions: In this study, we studied a novel chlorophylldeficient tree peony cultivar, which exhibited a yellow leaf phenotype, and performed the physiological characteristics and SSR analysis of the $y l l$ mutant. In conclusion, the lower chlorophyll and abnormal thylakoid structure in the $y l l$ mutant resulted in a lower $P_{\mathrm{N}}$, and the difference in DNA levels might be one of the causes of its mutation.

\section{References}

Berry J.A., Downton W.J.S.: Environmental regulation of photosynthesis. - In: Govindjee (ed.): Photosynthesis: Development, Carbon Metabolism, and Plant Productivity. Vol. II. Pp. 263-343. Academic Press, New York 1982.

Bogorad L.: Porphyrin synthesis. - Method. Enzymol. 5: 885-895, 1962.

Brestič M., Živčák M., Kunderlíková K. et al.: Low PSI content limits the photoprotection of PSI and PSII in early growth stages of chlorophyll $b$-deficient wheat mutant lines. Photosynth. Res. 125: 151-166, 2015.

Brestič M., Živčák M., Kunderlíková K., Allakhverdiev S.I.: High temperature specifically affects the photoprotective responses of chlorophyll $b$-deficient wheat mutant lines. Photosynth. Res. 130: 251-266, 2016.

Cao H., Li H., Miao Z. et al.: [The preliminary study of leaf-color mutant in Dendrobium officinale]. - J. Nucl. Agric. Sci. 31: 461-471, 2017. [In Chinese]

Chang Q.S., Chen S.M., Chen Y. et al.: Anatomical and physiological differences and differentially expressed genes between the green and yellow leaf tissue in a variegated chrysanthemum variety. - Mol. Biotechnol. 54: 393-411, 2013.

Chen H., Cheng Z.J., Ma X.D. et al.: A knockdown mutation of YELLOW-GREEN LEAF2 blocks chlorophyll biosynthesis in rice. - Plant Cell Rep. 32: 1855-1867, 2013.

Chen L., Yuan Q.Z.: [Study on Luoyang tourism climate comfort based on entropy method]. - J. Luoyang Normal Univ. 37: 20-25, 2018. [In Chinese]

Chen Q., Wang L.F., Su N. et al.: Photosystem 2 photochemistry and pigment composition of a yellow mutant of rice (Oryza sativa L.) under different irradiances. - Photosynthetica 46: 35-39, 2008.

Dei M.: Benzyladenine-induced stimulation of 5-aminoleuvulinic acid accumulation under various light intensities in levulinic acid-treated cotyledons of etiolated cucumber. - Physiol. Plantarum 64: 153-160, 1985.

Deng Y.M., Shao Q.S., Li C.C. et al.: Differential responses of double petal and multi petal jasmine to shading: II. Morphology, anatomy and physiology. - Sci. Hortic-Amsterdam 144: 19-28, 2012.

Dong H., Fei G.L., Wu C.Y. et al.: A rice virescent-yellow leaf mutant reveals new insights into the role and assembly of plastid caseinolytic protease in higher plants. - Plant Physiol. 162: 1867-1880, 2013.

Georgieva K., Fedina I., Todorova Maslenkova L., Peeva V.: Response of chlorina barley mutants to heat stress under low and high light. - Funct. Plant Biol. 30: 515-524, 2003.

Guo Q.: [Construction of genetic linkage map using SSR molecular markers in tree peony]. Master Thesis. Henan Univ. Sci Technol., Henan 2016. [In Chinese]

Hou X.G., Guo D.L., Cheng S.P., Zhang J.Y.: Development of thirty new polymorphic microsatellite primers for Paeonia suffruticosa. - Biol. Plantarum 55: 708-710, 2011.

Hu Z., Lian H.X., Peng L.P.: [Photosynthetic characteristics of a yellow-green rice mutant]. - Acta Bot. Yunnan. 3: 440-456, 1981. [In Chinese]

Hung C.Y., Xie J.H.: A comparison of plants regenerated from a variegated Epipremnum aureum. - Biol. Plantarum 53: 610-616, 2009.

Lei H.M., Nie Q., Liu R.X. et al.: [Photosynthetic characteristics of a mutant with yellow leaf in tobacco]. - Guizhou Agric. Sci. 38: 9-11, 2010. [In Chinese]

Li Q.Z., Zhu F.Y., Gao X.L. et al.: Young leaf chlorosis 2 encodes the stroma-localized heme oxygenase 2 which is required 
for normal tetrapyrrole biosynthesis in rice. - Planta 240: 701-712, 2014

Nagata N., Tanaka R., Satoh S., Tanaka A.: Identification of a vinyl reductase gene for chlorophyll synthesis in Arabidopsis thaliana and implications for the evolution of Prochlorococcus species. - Plant Cell 17: 233-240, 2005.

Ou L.J., Li W.J., Tian L.F. et al.: Photosynthetic characteristics of $\mathrm{C}_{4}$ trait in chlorina mutant of rice (Oryza sativa L.). Photosynthetica 46: 589-594, 2008.

Pan R.Z.: [Plant physiology]. Pp. 56-82. Higher Education Press, Beijing 2004. [In Chinese]

Rebeiz C.A., Mattheis J.R., Smith B.B. et al.: Chloroplast biosynthesis: Biosynthesis and accumulation of protochlorophyll isolated etioplasts and developing chloroplasts. - Arch. Biochem. Biophys. 171: 549-567, 1975.

Reumann S., Inoue K., Keegstra K.: Evolution of the general protein import pathway of plastids (review). - Mol. Membr. Biol. 22: 73-86, 2005.

Sakamoto W., Miyagishima S., Jarvis P.: Chloroplast biogenesis: Control of plastid development, protein import, division and inheritance. - Arabidopsis Book 6: e110, 2008.

Teng N.J., Wang J., Chen T. et al.: Elevated $\mathrm{CO}_{2}$ induces physiological, biochemical and structural changes in leaves of Arabidopsis thaliana. - New Phytol. 172: 92-103, 2006.

Terao T., Katoh S.: Antenna sizes of photosystem I and photosystem II in chlorophyll $b$-deficient mutants of rice. Evidence for an antenna function of photosystem II centers that are inactive in electron transport. - Plant Cell Physiol. 37: 307-312, 1996

Timmis J.N., Ayliffe M.A., Huang C.Y., Martin W.: Endosymbiotic gene transfer: Organelle genomes forge eukaryotic chromosomes. - Nat. Rev. Genet. 5: 123-135, 2004.

Wang C.X., Tian W.W., Tian M. et al.: [The preliminary study of xantha mutants in Oncidium]. - J. Nucl. Agric. Sci. 27: 1845-1852, 2013. [In Chinese]

Wang J.: [Development of EST-SSR primers and application in analysis of genetic relationships in tree peony]. Master Thesis. Henan Univ. Sci. Technol., Henan 2011. [In Chinese]

Wang L., Yue C., Cao H.L. et al.: Biochemical and transcriptome analyses of a novel chlorophyll-deficient chlorina tea plant cultivar. - BMC Plant Biol. 14: 352, 2014.

Wang Y., Zheng W., Zheng W.J. et al.: Physiological and transcriptomic analyses of a yellow-green mutant with high photosynthetic efficiency in wheat (Triticum aestivum L.). Funct. Integr. Genomic. 18: 175-194, 2018.

Wang Y.Q., Shi J.Q., Zhang T. et al.: [Characterization and candidate gene analysis of yellow-green leaf mutant ygll3 in rice (Oryza sativa)]. - Sci. Agr. Sin. 48: 4197-4208, 2015. [In Chinese]

Wu Z., Zhang X., He B. et al.: A chlorophyll-deficient rice mutant with impaired chlorophyllide esterification in chlorophyll biosynthesis. - Plant Physiol. 145: 29-40, 2007.
Wu Z.M., Zhang X., Wang J.L., Wan J.M.: Leaf chloroplast ultrastructure and photosynthetic properties of a chlorophylldeficient mutant of rice. - Photosynthetica 52: 217-222, 2014.

Yang D.L., Li S., Li M.F. et al.: Physiological characteristics and leaf ultrastructure of a novel chlorophyll-deficient chd6 mutant of Vitis vinifera cultured in vitro. - J. Plant Growth Regul. 31: 124-135, 2012.

Yang X.M., Wu X.L., Liu Y.F. et al.: [Analysis of chlorophyll and photosynthesis of a tomato chlorophyll-deficient mutant induced by EMS]. - Chin. J. Appl. Ecol. 29: 1983-1989, 2018. [In Chinese]

Yang Y.X., Chen X.X., Xu B. et al.: Phenotype and transcriptome analysis reveals chloroplast development and pigment biosynthesis together influenced the leaf color formation in mutants of Anthurium andraeanum 'Sonate'. - Front. Plant Sci. 6: 139-154, 2015.

Zhang K., Liu Z.Y., Shan X.F. et al.: Physiological properties and chlorophyll biosynthesis in a Pak-choi (Brassica rapa L. ssp. chinensis) yellow leaf mutant, pylm. - Acta Physiol. Plant. 39: 22, 2017.

Zhang L.J., Fan J.J.: [Plant Physiology Experiment Course]. China Agricultural University Press, Beijing 2007. [In Chinese]

Zhao C., Xu J., Chen Y. et al.: Molecular cloning and characterization of OsCHR4, a rice chromatin-remodeling factor required for early chloroplast development in adaxial mesophyll. - Planta 236: 1165-1176, 2012.

Zhao F.M., Zhang Y., Wu Y.R. et al:: Morphological and physiological analysis of narrow and striped leaf 1 (nsl1) mutant of rice (Oryza sativa L.) and the gene mapping. Chinese Sci. Bull. 59: 840-848, 2014.

Zhao S., Long W., Wang Y. et al.: A rice White-stripe leaf3 (wsl3) mutant lacking an HD domain-containing protein affects chlorophyll biosynthesis and chloroplast development. J. Plant Biol. 59: 282-292, 2016.

Zhou H.: [Discovery of genes associated with flowering time in tree peonies based on transcriptome comparison]. Doctoral Thesis. Beijing Forestry University, Beijing 2015. [In Chinese]

Zhu L.X., Zeng X.H., Chen Y.L. et al.: Genetic characterisation and fine mapping of a chlorophyll-deficient mutant (BnaC.ygl) in Brassica napus. - Mol. Breeding 34: 603-614, $2014 \mathrm{a}$.

Zhu X.J., Shang A.Q., Yang M.S. et al:: [Photosynthetic characteristics and leaf coloration mechanism of the next generation of golden Ulmus pumila]. - Acta Bot. Boreal.Occident. Sin. 34: 950-956, 2014b. [In Chinese]

Živčák M., Brestič M., Botyanszka L. et al.: Phenotyping of isogenic chlorophyll-less bread and durum wheat mutant lines in relation to photoprotection and photosynthetic capacity. Photosynth. Res. 139: 239-251, 2019.

(C) The authors. This is an open access article distributed under the terms of the Creative Commons BY-NC-ND Licence. 